\title{
Research on the Architecture of Urban Emergency System Based on GIS Zhe $\mathrm{Li}^{1, \mathrm{a}}$, Xiang Teng ${ }^{2, \mathrm{~b}}$ \\ ${ }^{1}$ College of International Exchange, Bohai University, Jinzhou, 121013, China \\ ${ }^{2}$ Basic Teaching and Research Institute, Bohai University, Jinzhou, 121013, China \\ a503412508@qq.com, byyq8369@163.com
}

Keywords: geographic information system; GIS; Urban emergency; system architecture; spatial Data; MVC

\begin{abstract}
Architecture technology is development model based on some sort of application, offers a wide range of software reuse to provide a unified software development kit for developers and model architecture. First, research the infrastructure consists of Core layer, organization layer, communication layer, executive layer and collaborative layer; Then, research the data structure that business layer department building and maintaining independently and shared with each section based on the spatial data structure of geographic information. Finally, research the MVC software architecture, describing the division of labor and cooperation among the Model, View and Controller. Application of this results, not only significantly improve the development efficiency, and the system has more advantage like clear structure, stable performance, easy maintenance and easy to transplant, etc.
\end{abstract}

\section{Introduction}

Urban emergency system is to bring public security, transportation, communications, electricity, air defense, municipal administration and other government departments into a unified scheduling command system, to deal with emergencies and offer to the public of the information system that urban social emergency rescue service, realize the unified command of across different regions and departments, rapid response and joint operations, provide powerful guarantee for urban public safety. With China's economic and social development, the rural population to cities, small and medium-sized cities population to concentrate in large cities, cities are becoming increasingly large. The city's infrastructure, service level is far can not adapt to the needs of growing population, and frequent incidents, the influence of the incident is sometimes disastrous. The incident with uncertainty, the disposal of uncertainty, the uncertainty of results, impact of uncertainty. Because there are a lot of information and many uncertain factors, the emergency management is very complicated.

Geographic information system (GIS) is a comprehensive discipline, combining with geography and cartography, remote sensing and computer science, is widely applied in different fields, as input, storage, query, analysis and display of Geographic data of a computer system.GIS map this unique visual effects and geographic analysis function and the general database operations together. GIS information visualization expression and a strong geographical spatial analysis function, show resources visualization query and decision analysis provides the effective working platform and technical support, to achieve the goals of urban emergency system provides the ideal technical support platform.

Software architecture is a series of abstract patterns, a sketch of a system, used to guide the design of the aspects of large Software systems. Software architecture's description of object is directly constitute a system of abstract component, the connection between the various components are clear and relatively detailed describe the communication between components [1]. In the implementation phase, these abstract components are divided into actual components such as class or object. In the field of object oriented, the connection between the components usually use interface to implement. According to the characteristics of urban emergency system is complicated 
and difficult to development, this article launches the research based on GIS, and provides overall architecture for system development.

\section{System Fundamental Architecture}

Urban emergency system, it is to point to accept the public in a city report an emergency or urgent ask for help, and integrate the city all kinds of emergency rescue force as well as the municipal public service resources, realize the launching, multi-sectoral, multi-level and cross-regional, called unity, unified command and joint operations, to achieve timely, orderly and efficiently carry out emergency rescue and disaster relief operations, city public security comprehensive relief system and integration technology platform. Urban emergency system for natural disasters, accidents disasters, public health events and social security incident report to the police, for help and complaints, receiving alarm and linkage police achieve unity, all within the administrative jurisdiction has the function of public emergency disposal unified command dispatching emergency linkage unit, for disposal between different departments within the jurisdiction of the administrative emergency, emergency, major events for communications and information security [2-4]. System infrastructure is shown in Fig. 1.

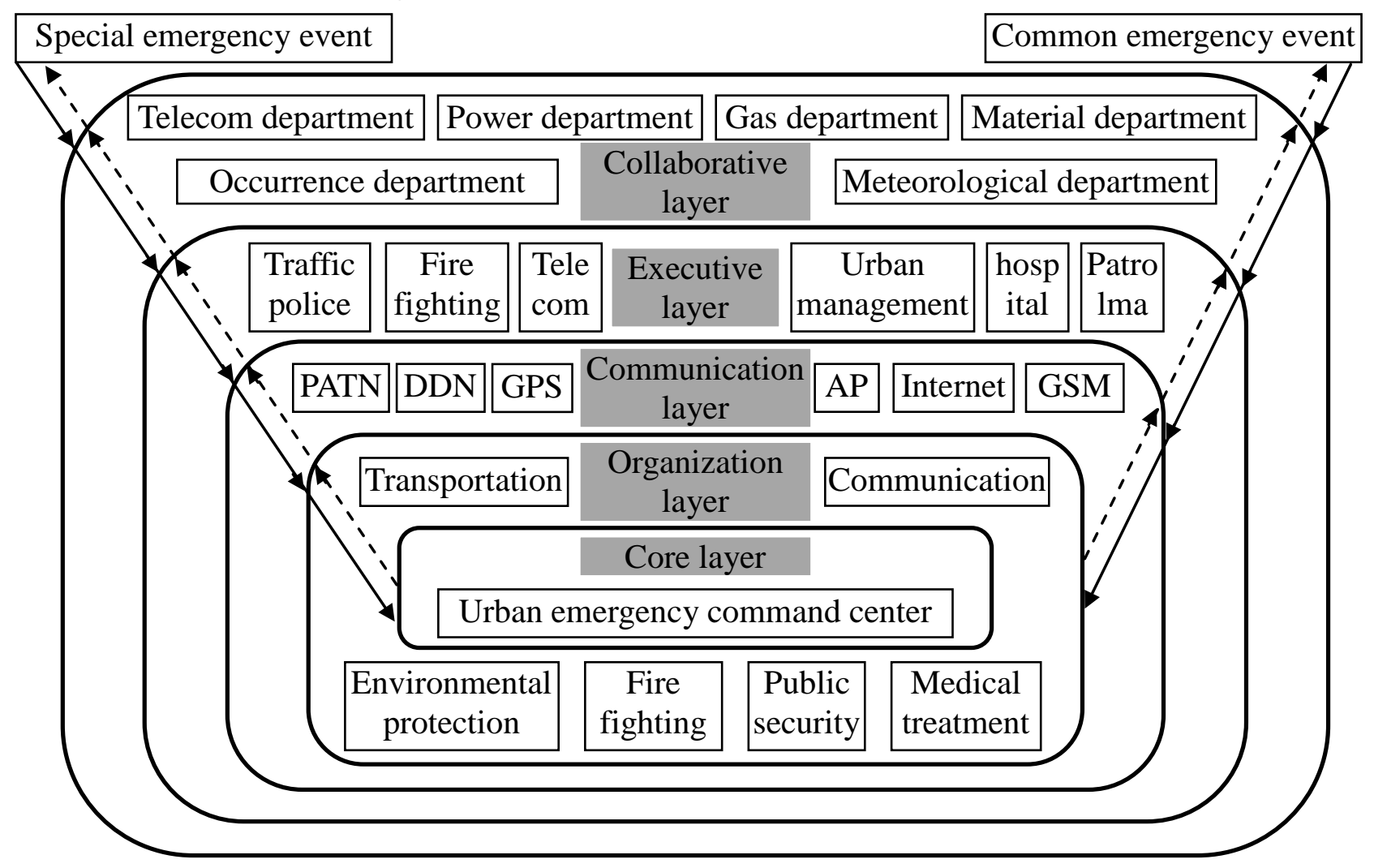

Fig. 1. Fundamental architecture on urban emergency system

As shown in Fig. 1 of the urban emergency system infrastructure consists of five layers: the Core layer, is a concentration of dispatching center, network center, communication center, monitoring center, data center, information center and distribution center as one of the central nervous system; Organization layer, made up of city operation and management of functional departments, complete the emergency disposal of resource allocation and scheduling; The Communication layer, to provide all kinds of wired and wireless Communication means to guarantee the Communication in the process of emergency; Executive layer, is the city emergency and emergency information receiver and gatherers and scheduling command, is the intermediate node of urban emergency management system; In the process of Collaborative layer, it is to point to to executive level emergency support of various functional departments or individuals. 


\section{Spatial Data Architecture}

Spatial data is used to indicate the location of the spatial entity, shape, size and its distribution information of data, used to describe the target from the real world, with positioning, qualitative characteristics, the relationship between time and space. Spatial data is a kind of point, line, face and entities such as the basic spatial data structure to represent the people rely on the data of the natural world.

Spatial data is the basis of the digital earth information, for the most part the function of digital earth will be based on the spatial data. Now the spatial data has been widely used in various industries, departments society, such as city planning, transportation, bank, aerospace, etc. With the development of science and society, people have increasingly realize the spatial data for the development of social economy, the importance of improving people's living standard, it also accelerated the pace of people to access and application of spatial data. Spatial data is a special type of data. Refers to all data with spatial coordinates, such as architectural blueprints, mechanical drawings and maps said into digital form that the computer can accept [5].

The most basic part of GIS is data, is the foundation of all GIS's function. Based on the original spatial data processing, display the contents of the real world. A good GIS geography information system, must be based on reliable data on geographical location, the location data can be through the sensor information acquisition, and then transferred to the geographic location information data processing center, including spatial data and attribute data, spatial data representation is represented by the object's location information, shape, size, etc. Urban emergency system of spatial data structure is shown in Fig. 2.

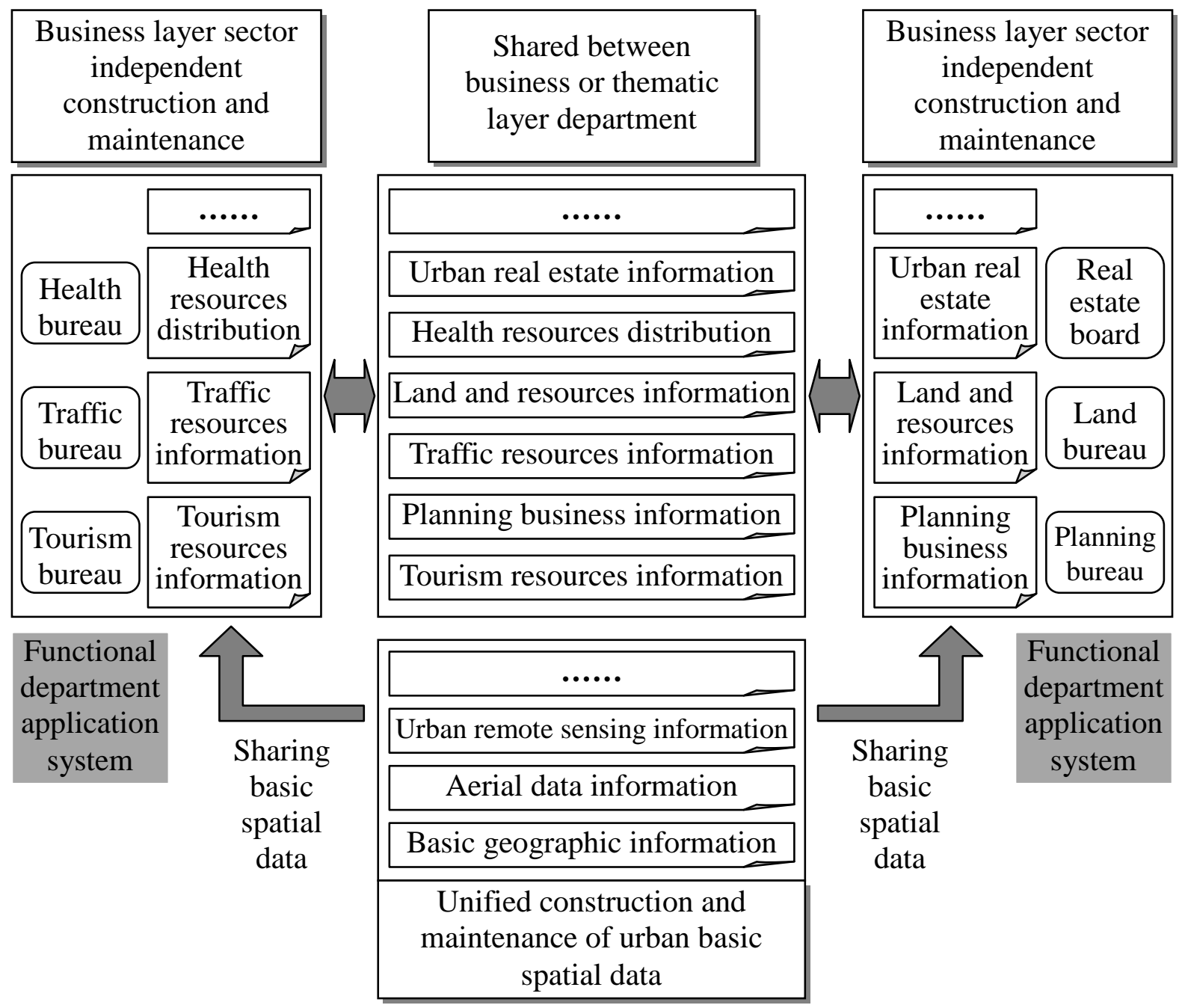

Fig. 2. Spatial data architecture on geographic information system

Usual spatial data management system should have the following five features: 
(1) Input data. The location data, statistical data and remote sensing data, processed into a computer can be expressed. And the graphic data, GPS data and attribute data to deal with different source data.

(2) Data editing. Including graphic editor and attributes. Graphical editing is mainly on the topology of the graphics object, graphics transformation, projection transformation, graphic splicing and error correction, etc.; Property editor is mainly combined with database with data.

(3) Management and preservation of data. To collect data for conservation and management, provide data query and retrieval and modification, etc. Through conservation and management of spatial data, spatial data and attribute data. Layering with space, and thematic data organization method of spatial data kept by GIS system management, attribute data shall be kept in a relational database management.

(4) Query and analysis. One is information retrieval, based on the corresponding space object space location information retrieval; Second, topology analysis, through the spatial characteristics of intersection, subtract, and merge operation, realize the analysis of the characteristic properties in space; Three is model analysis, $3 \mathrm{~d}$ model for a specific model, terrain height and network link analysis, etc.

(5) Visualization output and expression. To visual representation of geographical location information, facilitate intuitive feelings and analysis of the data, realize the human-computer interaction. Can be in relation to the zoom in and out of the graphic data. According to the needs of users, to subject of geography information data statistics, analysis and data display icon.

\section{MVC Software Architecture}

MVC (Model View Controller), with a method of separation of business logic, data, and display interface organization code, the business logic gathered on a widget, in improving and personalized custom interface and user interaction at the same time, do not need to rewrite the business logic. MVC's purpose is to increase the rate of code reuse, reduce data expression, data description and application of the coupling of the operation. At the same time, to improve the software maintenance, repair, extensibility, flexibility and encapsulation [6, 7].

The modes of data from a variety of separated under the control of access and data, improve the design of the distributed system. The MVC design pattern consists of three parts: the model is the application object, no user interface; View it on the screen display, on behalf of the flow to the user's data; Controller to define the user interface for the user to enter the way of response, be responsible for the operation of the user's action into according to the Model. The Model by updating data to reflect the changes of the View. MVC's software architecture is shown in Fig. 3.

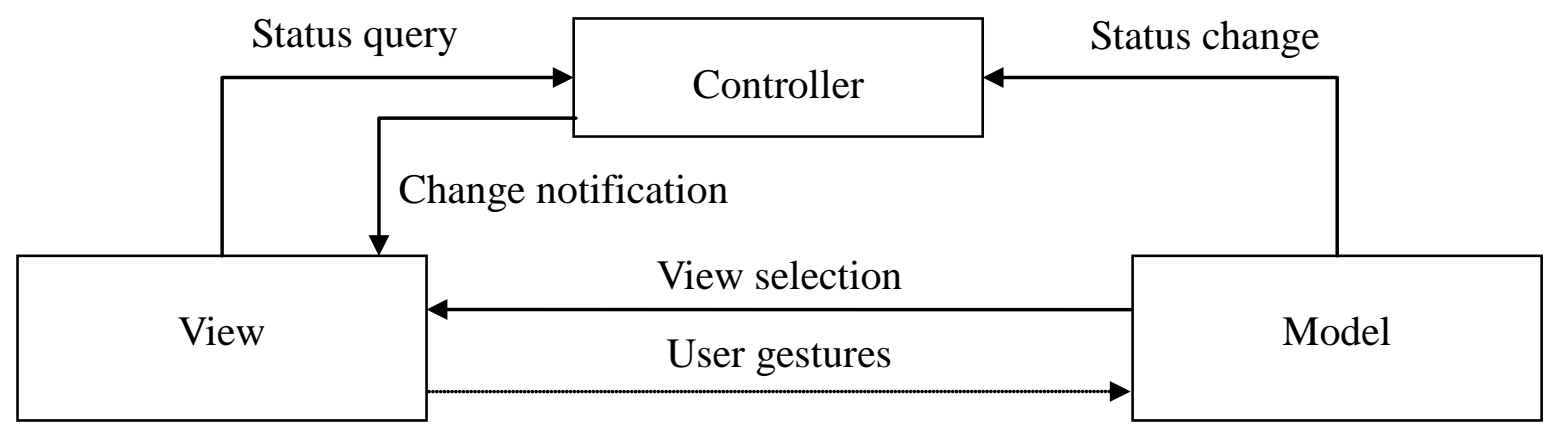

Fig. 3. Software architecture on MVC

There are both division of labor and collaboration among Model, View and Controller, a detailed description as shown in Table 1.

In the process of system development, from the perspective of a high level of an object can be divided into three categories: a class is responsible for the display of objects, a class of object contains business rules and data, there are those who receives the request, control business objects to complete the request. These applications show is often need to transform, such as web page style and tone, and need to display the content of the content display mode, etc. The business rules and 
data is relatively stable. Said display object View, therefore, often need to change, according to business rules and data object Model to a relatively stable, the said control Controller was the most stable.

Table 1. Division and Coordination of MVC

\begin{tabular}{|c|c|c|c|}
\hline Content & Model (M) & View (V) & Controller (C) \\
\hline Division & $\begin{array}{l}\text { Function of abstract } \\
\text { system application } \\
\text { State of encapsulation } \\
\text { system } \\
\text { Provide ways to use the } \\
\text { function of system } \\
\text { Storage and consistency } \\
\text { of management data } \\
\text { Notify relevant part when } \\
\text { data changes }\end{array}$ & $\begin{array}{l}\text { Expression of Abstract } \\
\text { system } \\
\text { Show data of } \\
\text { contraposing user } \\
\text { Maintain consistency } \\
\text { with the Model data }\end{array}$ & $\begin{array}{l}\text { Semantic mapping of } \\
\text { abstract user and system } \\
\text { matter } \\
\text { Translate user input into } \\
\text { system matter } \\
\text { Choose the appropriate data } \\
\text { based on user input and } \\
\text { context }\end{array}$ \\
\hline $\begin{array}{l}\text { Coordin } \\
\text { ation }\end{array}$ & $\begin{array}{l}\text { Notify View when system } \\
\text { data changes } \\
\text { Can be retrieved data by } \\
\text { View } \\
\text { Provide the operation } \\
\text { ways to Controller }\end{array}$ & $\begin{array}{l}\text { Show Model to user } \\
\text { Update the data } \\
\text { concerned when data } \\
\text { changed by Model } \\
\text { Submit user input to } \\
\text { Controller }\end{array}$ & $\begin{array}{l}\text { Turn user input into system } \\
\text { behavior of Model } \\
\text { Choose the appropriate } \\
\text { View based on user input } \\
\text { and working results of } \\
\text { Model }\end{array}$ \\
\hline
\end{tabular}

\section{Conclusion}

Computer technology, geographic information system technology, global positioning system (GPS) technology, network technology, database technology and communication technology, such as technology , for the urban public emergency response system provides a new solution. Efficient urban emergency system should be guided by the scientific contingency plans, with abundant information and the visualization of geographic information data as the foundation, through the information integration technology that unique geographic information and the GIS spatial analysis ability, combined with GPS positioning technology and characteristic of emergencies, with strong scientific and practical platform for the emergency command information [8]. This article research results in the successful application of urban emergency system, not only significantly improve development efficiency, but the developed software has clear structure, stable performance, easy maintenance and easy to transplant, and other advantages, have important practical significance to improve the level of city emergency, to other information platform development at the same time also can provide reference.

\section{Acknowledgement}

This work is supported by 2014 annual social science planning fund project of Liaoning province (L14DGL043): Improving to countermeasures on urban emergency government emergency management capacity.

\section{References}

[1] X. Y. Zhang, L. S. Zhao, "Research on Framework Structure for Sports Logistics Information Platform," Logistics Technology, vol. 36, no. 6, pp. 283-285, 2015.

[2] L. H. Jiang, L. Wang, "Design of Urban Emergency Response System Based on Digital Trunking," Journal of Changsha University, vol. 29, no. 5, pp. 61-62, 2015. 
[3] Y. Qi, "The Research and Implementation of Emergency Management Information Reporting System Based on GIS and Knowledge Base," Master's degree of Hunan, 2014.

[4] C. F. Tian, "Research on urban emergency management system based on GIS," Master's degree of University of Electronic Science and Technology, 2010.

[5] Madjid Tavana, Weiru Liu, Paul Elmore, et al, "A practical taxonomy of methods and literature for managing uncertain spatial data in geographic information systems," Measurement, vol. 81, no. 3, pp. 123-162, 2016.

[6] Xuan Yin, Wei Zheng, Ming Zhang, et al, "A modularized operator interface framework for Tokamak based on MVC design pattern," Fusion Engineering and Design, vol. 89, no. 5, pp. 628-632, 2014.

[7] Dragos-Paul Pop, Adam Altar, "Designing an MVC Model for Rapid Web Application Development," Procedia Engineering, vol. 69, no. 1, pp. 1172-1179, 2014.

[8] F. M. Lin, "The application of GIS technique in urban public emergency command system," Geomatics \& Spatial Information Technology, vol. 32, no. 3, pp. 31-33, 2009. 